\title{
Exploratory Compatibility Regularity of Chinese Medicinal Prescription in Treating Osteoarthritis
}

\author{
Zhou Qiao ${ }^{1 *}$, Liu Jian², Xin Ling ${ }^{2}$, Song Qian ${ }^{1}$, Guo Jin-chen ${ }^{1}$ and Huang Dan ${ }^{1}$
}

${ }^{1}$ Department of Postgraduates, Anhui University of Traditional Chinese Medicine, Hefei, China

${ }^{2}$ Department of Rheumatology, The First Affiliated Hospital of Anhui University of Traditional Chinese Medicine, Hefei, China

"Corresponding author: Qiao Z, Department of Postgraduates, Anhui University of Traditional Chinese Medicine, Hefei-230038, China; Tel: 13739240816; E-mail: 780149795@qq.com

Rec date: 02 Feb 2017, Acc date: 20 July 2017, Pub date: 27 July 2017

Copyright: @ 2017 Qiao Z, et al. This is an open-access article distributed under the terms of the Creative Commons Attribution License, which permits unrestricted use, distribution and reproduction in any medium, provided the original author and source are credited.

\begin{abstract}
Background: Traditional Chinese medicine (TCM) is increasingly widely used as a complementary approach for the treatment of osteoarthritis. In this paper, we examined the implicit prescription patterns behind the Chinese medicinal formulae by using association rules mining and clustering analysis, so as to explore the compatibility regularity of Chinese medicinal prescription in the treatment or control of osteoarthritis.

Methods: This study was based on a participant survey conducted in First Affiliated Hospital of Anhui University of Chinese Medicine. The Chinese medicinal prescriptions of inpatients with osteoarthritis were from June 2012 to June 2016. TCM was listed according to the selected medicinal formulae and the added information was organized to establish a database. The association rules and clustering are conducted on SPSS Modeler 14.2. An initial statistical analysis was carried out to categorize the herbs according to their medicinal types, dosage, natures, flavors, channel tropism, and functions. Based on the categorization, the frequencies of occurrences were computed. At the same time, the relationship between TCM and immune and inflammatory markers was analyzed.

Results: The main features from the formulae data are: (1) Warm or cold herbs in the Five Properties category are the most frequently prescribed in the 3609 medicinal formulae. Sweet or bitter herbs in the Five Flavors category in which affinity to the Spleen meridian also are the most frequently prescribed in the 3609 medicinal formulae. (2) Herbs with blood-activating and stasis-resolving, tonifying and replenishing medicinal, water-disinhibiting damppercolating, heat-clearing and detoxicating or expelling wind-damp functions that are frequently prescribed; (3) Association rule analysis extracted three frequently used pairs of herbs, which are Red flower and Tao Ren, Pu Gong Ying and Bai Hua She She Cao, Fu Ling and Ze Xie. (4) Association rule between TCM and Immunoinflammation Index showed that $\mathrm{Du}$ Huo and Chen $\mathrm{Pi}$ was associated with increased super oxide dismutase enzyme. Mai Ya and Dan Shen was associated with decreased high-sensitivity C-reactive protein, erythrocyte sedimentation rate, complement $\mathrm{C} 3$, complement $\mathrm{C} 4$, and $\lg (\mathrm{A})$. Mai $\mathrm{Ya}$ and $\mathrm{Fu}$ Ling were associated with decreased $\lg \mathrm{M}$.
\end{abstract}

Conclusions: The results indicate that there is a close relationship between osteoarthritis and spleen dysfunctions. These prescriptions focus on herbs that replenishing qi and fortifying the spleen and blood-activating and stasis-resolving. It can also dry dampness to fortify the spleen and clear heat and detoxicating. Meanwhile, he use of tonifying and replenishing herbs and spleen-strengthening herbs also forms a basic pattern of the prescriptions.

Keywords: Traditional Chinese medicine; Osteoarthritis; Herbs

\section{Background}

Osteoarthritis (OA) has done much harm to the elderly in their physical and mental health. It is the main cause of the elderly movement disorders, affecting the quality of life of millions of people around the world. The exact pathogenesis and etiology remains unclear [1]. Epidemiological study found that northern and eastern provinces in China had the lowest OA morbidity (5.4\% and 5.5\%, respectively). The prevalence rate in the northeastern region was $7.0 \%$. With Central and South regions to be $7.8 \%$, Northwest regions to be $10.8 \%$. The highest prevalence rate was in the southwestern region (13.7\%) [2]. The prevalence of $\mathrm{OA}$ increases gradually with the increasing age. According to statistics, the incidence of osteoarthritis or joint pain and other symptoms was $78.5 \%$ and $90 \%$ for people over 60 and 90 respectively [3,4].

Nowadays, the standard therapies for osteoarthritis include nondrug therapy and drug therapy. Non-drug therapy includes patient education and self-conditioning, Surgical treatment is applicable to those with severe functional impairment. Chinese medicine is a complete medical system with that has been in continuous practice for over 3000 years, during which time it has been widely used for OA treatment. The disease has a certain degree of disability. In the United States, osteoarthritis is the second leading cause of loss of work ability in men over 50. Research in China found that the incidence of 15 to 24 years old was $10 \%$, which goes up to $88 \%$ for people older than 55 . Most importantly, the disease can cause a disability rate as high as $53 \%$ [5]. 
Recently, the use of natural Chinese herb medicine for osteoarthritis is receiving more and more attention from the public. In traditional Chinese medicine (TCM), the treatment and prevention of osteoarthritis is a holistic approach in multi-level and through multichannel. TCM differs from Western medicine, which adopts ways to block a single transfer in a particular process. In comparison, Chinese medicine adopts an overall therapeutic approach to improve the immune system, and to strengthen the body's susceptibility to diseases. Meanwhile, Chinese medicine also aims at reversing drug resistance and improving quality of life. Therefore, these unique advantages have gradually made the Chinese medicinal approach in combating osteoarthritis the research focus for both the local and overseas scholars [6,7]. TCM Unit Therapy of Invigorating Spleen has a prominent curative effect on the improvement of life quality and cardiopulmonary function of patients with knee osteoarthritis, hose immunological mechanism also has been explored by animal experiments [8]. Our results demonstrate that the Chinese medicine Xin Feng capsule (XFC), which strengthens and replenishes qi and resolves dampness and unblocks channels, can significantly decrease joint pain, swelling, and tenderness. Cheng yuan-yuan [9] found that the XFC can decrease Mankin scores of cartilage and improve cardiopulmonary function of KOA rats. Its mechanism may be enhancing BTLA-HVEM negative co-stimulatory signals, inducing Treg immune tolerance, up-regulating interleukin-4 (IL-4), ownregulating interleukin-17 (IL-17), tumor necrosis factor $\beta \mathrm{u}$ (TNF- $\beta 1$ ), hen to inhibit abnormal inflammatory immune response. Xinfeng capsule [10] can significantly increase SF-36 scores, BTLA and interleukin-10(IL-10) levels, while decreased Lequesne MG, symptom classify quantization scores, interleukin-1 $\beta($ IL-1 $\beta)$ and malondialdehyde (MDA) levels. Xinfeng capsule [11] can inhibit the abnormal activation of the NF- $\mathrm{BB}$ signaling pathway, raise the level of interleukin-10(IL-10), reduce the expression of interleukin-1(IL-1), tumor necrosis factor $-\alpha$ (TNF- $\alpha), 50,65, A K 1$ and soon, nd reduce the abnormal inflammatory immune response so as to achieve the purpose of delaying and inhibiting the production of blood stasis, reduce joint disease, relieve the symptoms of joint pain and stiffness, eventually improve the patient's quality of life.

Traditional Chinese medicine prescription is under the guidance of the theory of Chinese medicine, through drug compatibility, avoiding weaknesses, adjusting the bias, the toxicity, enhancing or changing its original role, o eliminate or alleviate its adverse effects, o that the characteristics of the drugs are more and more in line with the needs of the disease. According to TCM theories, pharmacological and pharmacodynamic relationship exists among herbs, which are deemed as Chinese medicinal compatibility. The compatibility of Chinese herb medicine has particular rules and patterns. In Chinese medicinal database, here are over ten thousand medicinal formulae which include complicated information. However, a well-established and orderly system for organizing the information of Chinese medicinal formulae does not exist. This implies that a large amount of implicit prescription patterns behind the formulae have not been fully disclosed.

Association rule mining was first proposed by Agrawal [12]. And is used to deal with transactional data set. It has become a very important research method in data mining [13]. Apriori algorithm is a classical method of association rule mining. Its core is a kind of recursion based on frequent set theory. The advantages of this algorithm are that the principle is simple. The program is simple and easy to understand. The disadvantage is that the complexity is very high. Since then, aiming at the shortcomings of Apriori algorithm, varieties of improved algorithms are presented to improve the efficiency. Almost all of the existing improved algorithms are designed to improve operational efficiency. Its calculation object is still a single database [14].

\section{Materials and Methods}

\section{Diagnostic criteria}

All patients had a diagnosis of OA based on the revised criteria of the American Rheumatism Association (ACR) 1995 and the Chinese Society of Rheumatology 2003[15]. Eligible patients were those aged 40 to 75 years old who met the diagnostic criteria of Western medicine.

\section{Exclusion criteria}

We eliminated patients who:1) not meeting the Western diagnostic criteria for disease; 2) having combined disease of the circulatory system, respiratory system or hematopoietic system; 3) were either younger than 40 years old or older than 75 years old; 4) were pregnant women or nursing mothers; 5) other patients that were not suitable for the clinical trial(for example).

\section{Clinical data}

The data of patients with knee osteoarthritis are collected from June 2012 to June 2016. It comes from Department of Rheumatology, he First Affiliated Hospital of Anhui University of Chinese Medicine. The following indexs are selected: Immunoglobulin $\mathrm{A}(\operatorname{Ig} \mathrm{A})$, immunoglobulin $M(\operatorname{IgM})$, immunoglobulin $\mathrm{G}(\operatorname{IgG})$, complement $\mathrm{C} 3$, complement $\mathrm{C} 4$, Erythrocyte sedimentation rate(ESR), high sensitive C-reactive protein(hs-CRP) and superoxide dismutase(SOD). These patients have immune inflammation and metabolic index before and after the treatment.

\section{Data preprocessing}

The values of ESR, s-CRP, IgA, IgG, IgM, complement C3, complement $\mathrm{C} 4$ after treatment were determined to be "1", unchanged or increased to be " 0 "; the value of SOD was set at " 1 ", unchanged or decreased to " 0 "; (Note: we determined the variables to be" 1 " if the changes of indicators after treating help improving disease. On the contrary, to be " 0 " if not conducive to reduce disease.

The name of the herbs are from The Dictionary of Medicinal Plant [16] and the traditional Chinese pharmacology [17]. In this case, we can ensure the unity of traditional Chinese medicine name and prevent the same TCM from a different name.

The specification of TCM classification: The drugs of this study were classified according to the traditional Chinese pharmacology by Huang Zhao-sheng, dividing into 21 categories.

\section{Statistical analysis}

Classical association rules data mining algorithm Apriority algorithm is more and more widely used in the medical field $[18,19]$. Through the analysis and mining of data by association rules, medical data can be used to a greater extent. By analyzing the correlation between data, it provides a convincing reference for clinical diagnosis and treatment, and meets the requirement of evidence-based medicine system [20]. In order to analyze and excavate prescriptions of traditional Chinese medicine prescriptions for the treatment of osteoarthritis. In this study, the complete prescription medication 
Citation: Qiao Z, Jian L, Ling X, Qian S, Jin-chen G, et al. (2017) Exploratory Compatibility Regularity of Chinese Medicinal Prescription in Treating Osteoarthritis . Altern Integr Med 6: 244. doi:10.4172/2327-5162.1000244

Page 3 of 8

records of patients admitted to our hospital from June 2012 to June 2016 for rheumatoid arthritis were analyzed by association rule of Apriority algorithm, minimum support is set to 0.2 , indicating that the study of single drug in the study prescriptions appear more than $20 \%$. The minimum confidence is set to 0.7 , indicating that the study of related drugs on $A \rightarrow B$, using $A$ after the use of $B$ associated with the minimum probability of control in more than $70 \%$.

Based on Pharmacopoeia of the People's Republic of China, the ingredients of Chinese medicine were listed according to the selected medicinal formulae and were organized to establish a database. The computing soft-ware Microsoft ACCESS was used as a storage tool, and then the SPSS Modeler 14.2 Data Mining System was used as a platform to analyze the frequency and the association rules of the prescription patterns. An initial statistical analysis of the database was carried out to categorize the herbs according to their medicinal types and dosage, natures, flavors, channel tropism, and functions. The frequencies of occurrence and use were then computed based on the categorization. In addition the associations between different functions of Chinese herbs from the formulae were also examined using the association rules mining.

\section{Results}

\section{Associations between five properties and five flavors from 332 herbs prescribed in 3609 formulae}

The 332 herbs were categorized according to the Five Properties and Five Flavors (Figures 1 and 2). Based on the Five properties and Five flavors theory of TCM, erbs with a warm (122 herbs, 36.75\%) or cold (123 herbs, $37.05 \%$ ) were most frequently prescribed in terms of the occurrence frequency. Herbs with a neutral (appeared 3606 times, 99.92\%), warm (appeared 3593 times, 99.56\%), and cold (appeared 3318 times, 91.94\%) nature were the top three prescribed herbs in terms of the frequency of use. According to the Five Flavors (Figure 2) herbs that were sweet (appeared 3608 times, 99.97\%) or bitter (appeared 3606 times, 99.92\%) were the top two prescribed herbs in terms of both the frequencies of occurrence and use.

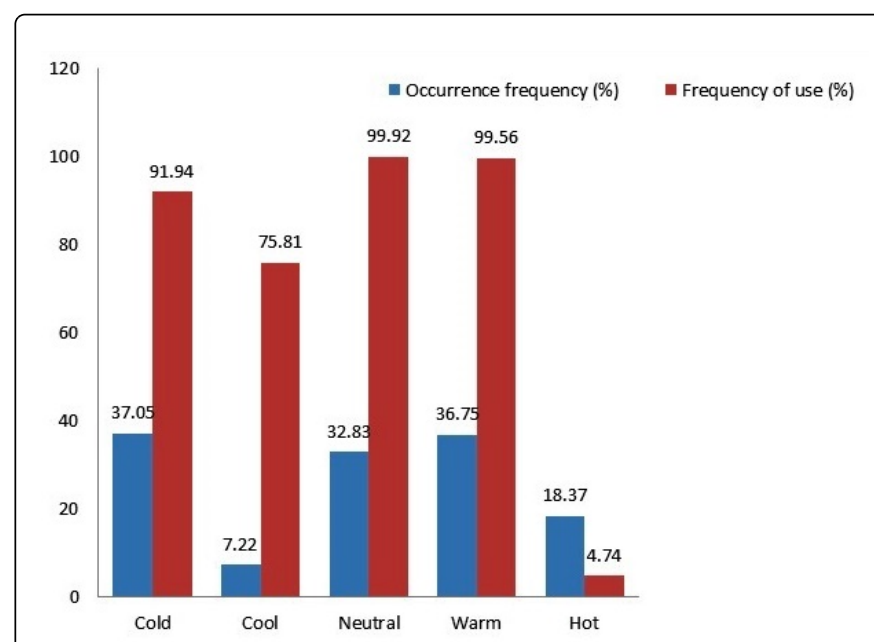

Figure 1: Association of the herbs from 332 formulae with Five Properties ( $\mathrm{N}=332$ in 3609 formulae).
Occurrence frequency=number of herbs belonging to each category/total number of recorded herbs (i.e. 332); Frequency of use=number of formulae recording the use of the herbs/total number of selected formulae (i.e.: 3609 ).

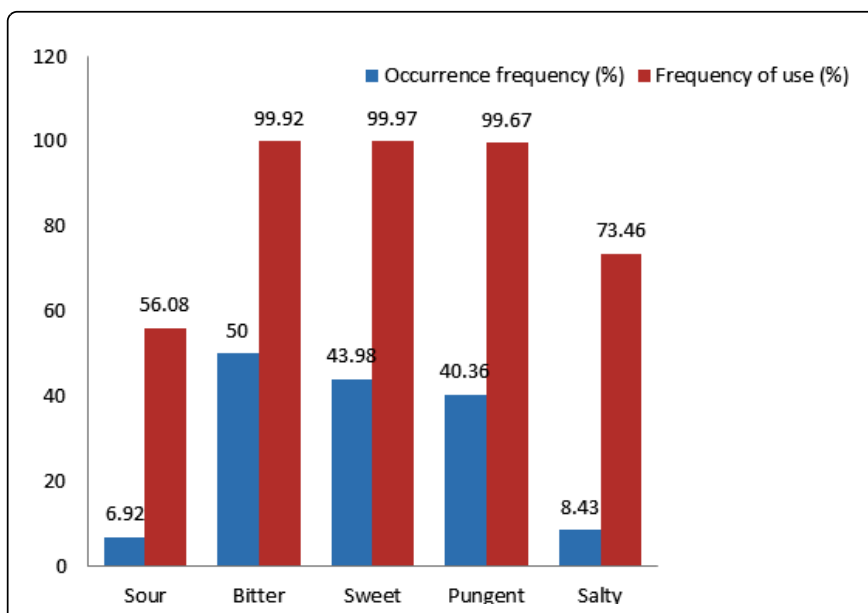

Figure 2: Association of the herbs from 332 formulae with Five Flavors ( $\mathrm{N}=332$ in 3609 formulae)

Occurrence frequency=number of herbs belonging to each category/total number of recorded herbs (i.e. 332); Frequency of use=number of formulae recording the use of the herbs/total number of selected formulae (i.e.: 3609)

Association between channel tropism theories from 332 herbs prescribed in 3609 formulae

The results of the association analysis based on channel tropism theory among 332 herbs (Figures 3 and 4) showed that the top two most frequently prescribed herbs have high affinity to the spleen and liver. The herbs that have high affinity to the spleen channel were used most frequently (frequency of use $=99.97 \%$ ).

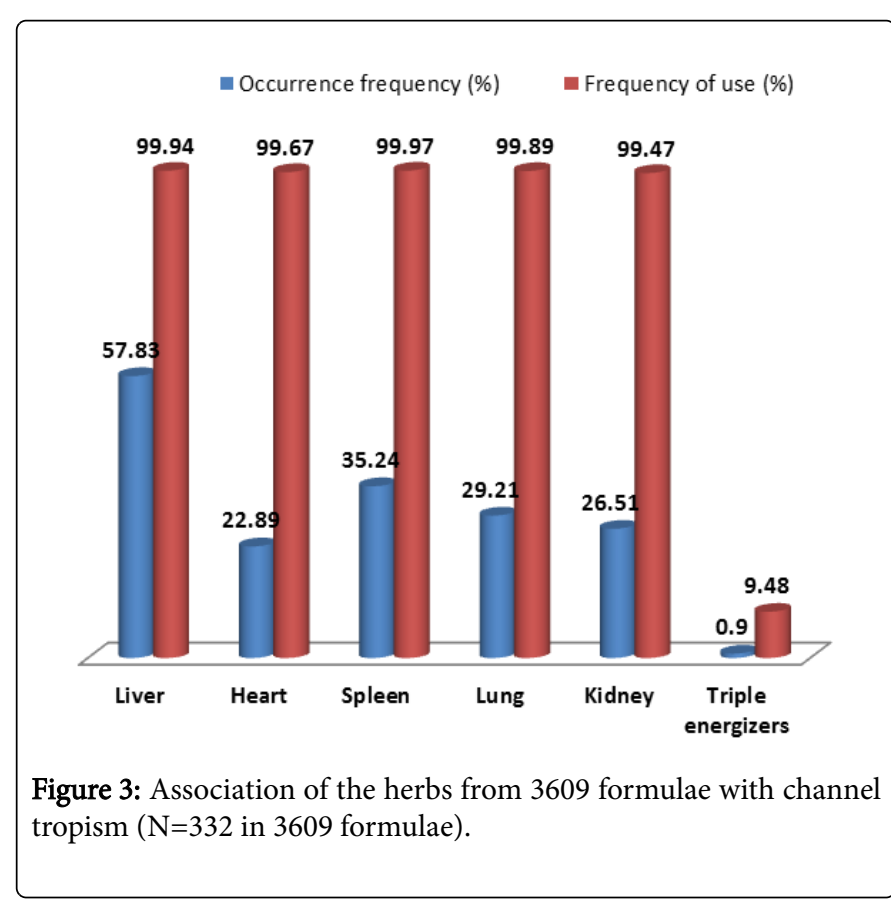




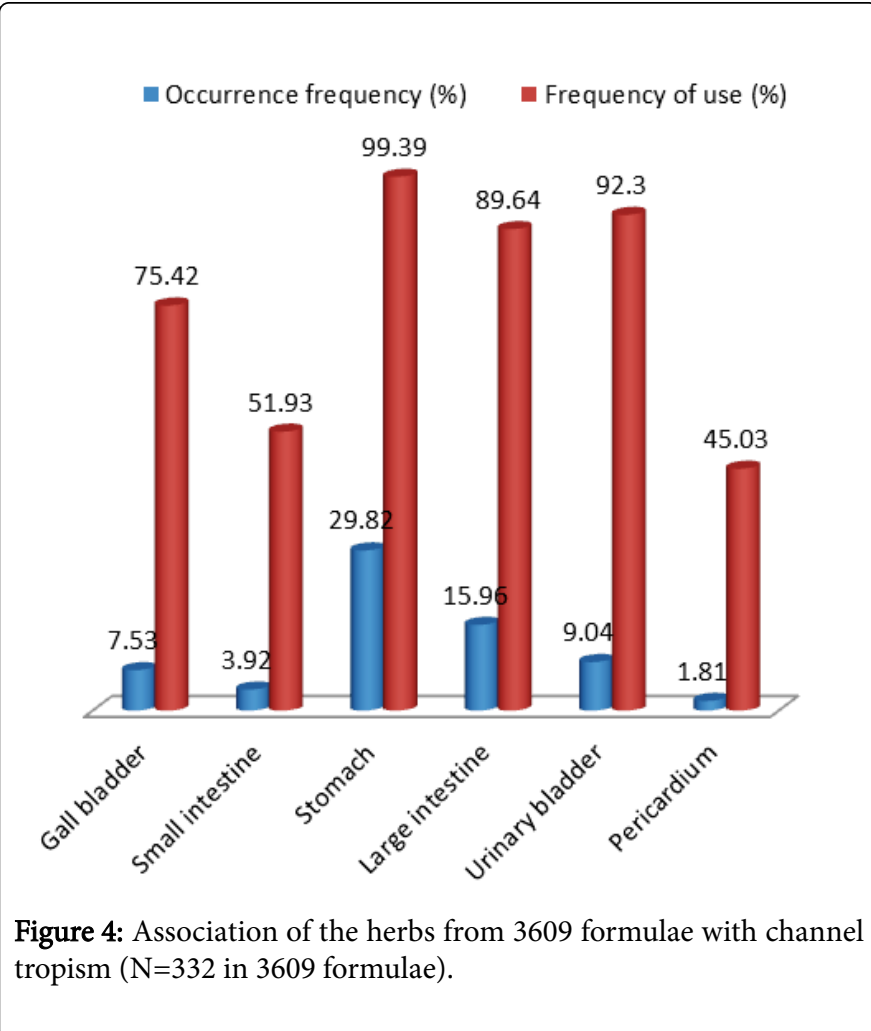

Occurrence frequency=number of herbs belonging to each category/total number of recorded herbs (i.e.: 332); Frequency of use $=$ number of formulae recording the use of the herbs/total number of selected formulae (i.e.: 3609).

\section{Frequency distribution of a single herb prescribed in Chinese medicinal formulae}

There are 3609 medicinal formulae with a total of 332 herbs included in this study. The total cumulative occurrence of 332 herbs appearing in 3609 formulae was 264047 times. The following is the top 22 herbs:

- Principal function in drugs for blood-activating and stasisresolving, including: Dan Sheng, Zhong Hua, Cao Ren, i XueTeng, Liu Xi

- Principal function in drugs for tonifying and replenishing, including: Gan Cao, Zhan Yao, u Zhong, a Zhu, Quang Qi, Bai Zhu

- Principal function in drugs for water-discharging and dampclearing, including: Fu Ling, i Yi Ren, e Xie

- Principal function in drugs for heat-clearing and detoxicating, including: Pu Gong Ying, ai Hua She She Cao

- Principal function in drugs for wind-dampness dispelling, including: Wei Ling Xian, i Xian Cao, u Huo

- Principal function in drugs for qi-regulating, including: Chen Pi

- Principal function in drugs for digestive, including: Mai Ya

- Principal function in drugs for exterior-releasing, including: Gui Zhi

- Principal function in drugs for dampness-resolving, including: $\mathrm{Hou} \mathrm{Pu}$

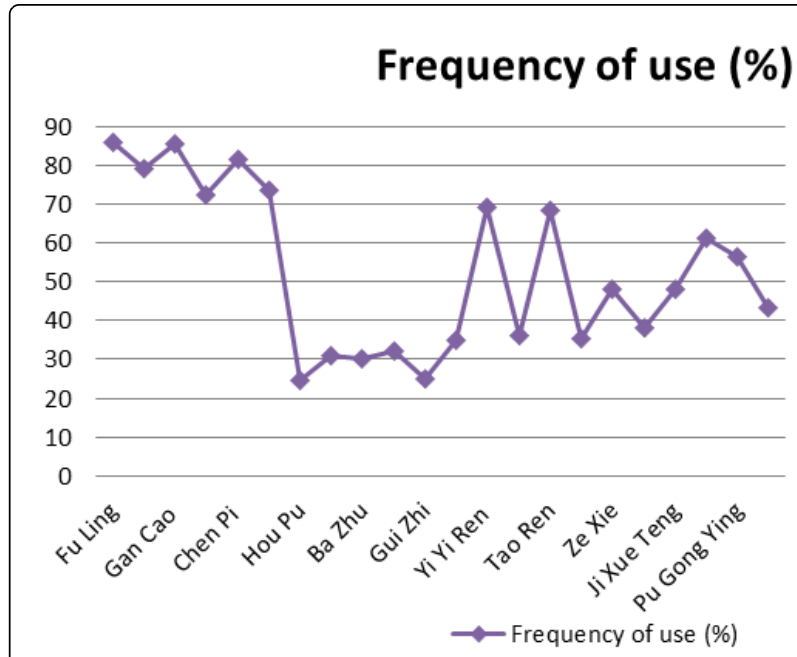

Figure 5: The top 22 herbs being used among the 332 herbs of the formulae in frequency.

Frequency of use=number of formulae recording the use of the herbs/total number of selected formulae (Table 1).

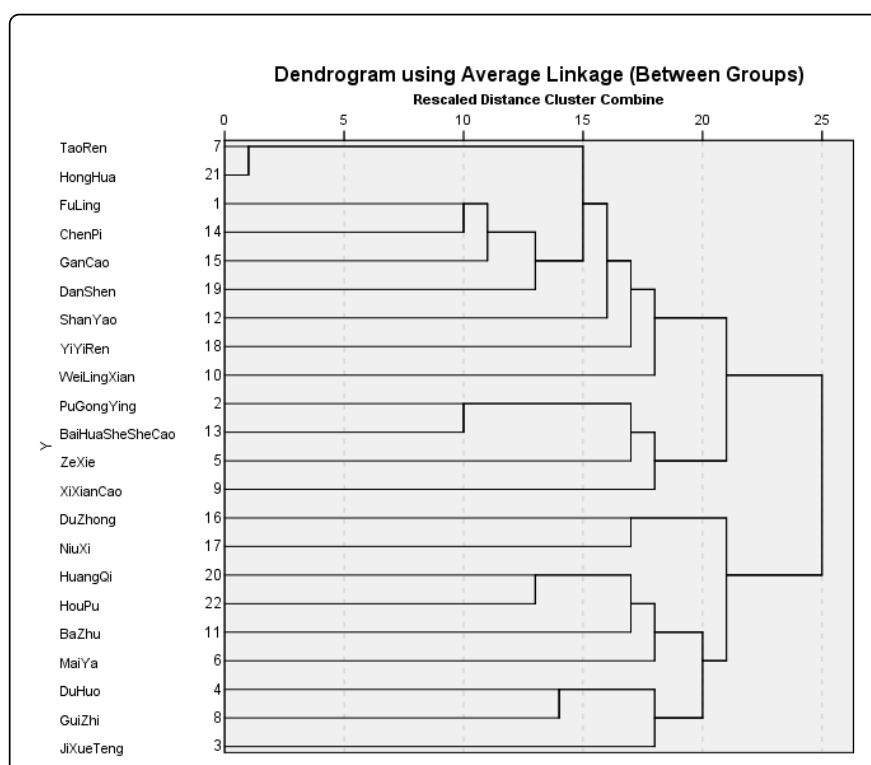

Figure 6: Cluster analysis of the tree.

Herbs with blood-activating and stasis-resolving, tonifying and replenishing, water-discharging and damp-clearing, eat-clearing and detoxicating, wind-dampness dispelling functions appeared to be most frequently prescribed for the treatment of osteoarthritis (Table 2).

The top three functions included herbs with blood-activating and stasis-resolving, tonifying and replenishing, and water-discharging and damp-clearing functions. 
Citation: Qiao Z, Jian L, Ling X, Qian S, Jin-chen G, et al. (2017) Exploratory Compatibility Regularity of Chinese Medicinal Prescription in Treating Osteoarthritis . Altern Integr Med 6: 244. doi:10.4172/2327-5162.1000244

Page 5 of 8

\begin{tabular}{|l|l|l|l|}
\hline Cluster & No. of members & Herbs & Main efficacy \\
\hline 1 & 2 & Tao Ren, ong Hua & blood-activating and stasis-resolving \\
\hline 2 & 2 & Chen Pi, u Ling & fortify the spleen and drain dampness \\
\hline 3 & 2 & Pu Gong Ying, ai Hua She She Cao & clearing away heat and toxic materials \\
\hline 4 & 2 & Du Zhong, iu Xi & restoring vital energy \\
\hline 5 & 3 & Huang Qi, ou Pu, ai Zhu & tonify qi and replenish blood \\
\hline 6 & 3 & Du Huo, ui Zhi, i Xue Teng & wind-dampness dispelling \\
\hline Frequency distribution of categorized herbs according to their functions & \\
\hline
\end{tabular}

Table 1: Results of cluster analysis.

\begin{tabular}{|c|c|c|c|c|c|}
\hline No & Functions & $\begin{array}{l}\text { Number of herbs occurrences in } \\
\text { various formulae }\end{array}$ & $\begin{array}{l}\text { Occurrence frequency } \\
(\%)\end{array}$ & $\begin{array}{l}\text { Number of formulae use } \\
\text { of the herbs }\end{array}$ & Frequency of use (\%) \\
\hline 1 & $\begin{array}{l}\text { Blood-activating and stasis- } \\
\text { resolving }\end{array}$ & 53440 & 20.23 & 28 & 8.43 \\
\hline 2 & Tonifying and replenishing & 48904 & 18.52 & 46 & 13.86 \\
\hline 3 & $\begin{array}{l}\text { Water-discharging and damp- } \\
\text { clearing }\end{array}$ & 37980 & 14.38 & 20 & 6.02 \\
\hline 4 & Heat-clearing and detoxicating & 34353 & 13.01 & 54 & 16.27 \\
\hline 5 & Wind-dampness dispelling & 30117 & 11.41 & 26 & 7.83 \\
\hline 6 & Qi-regulating & 15514 & 5.88 & 16 & 4.82 \\
\hline 7 & Digestive & 9462 & 3.58 & 6 & 1.81 \\
\hline 8 & Exterior-releasing & 8028 & 3.04 & 24 & 7.23 \\
\hline 9 & Dampness-resolving & 3590 & 1.36 & 7 & 2.11 \\
\hline
\end{tabular}

Table 2: The top 9 kinds of function herb among the 332 herbs of the formulae in frequency.

Association rules mining were applied to investigate the associations between pairs of herb functions from the formulae, and to examine the Chinese medicinal compatibility patterns. The minimum support of 0.2 and the minimum confidence of 0.7 were specified.

\begin{tabular}{|c|c|c|c|}
\hline After item & Before Item & Support (\%) & Confidence (\%) \\
\hline Tonifying and replenishing medicinal & Water-disinhibiting damp-percolating medicinal & 88.72 & 98.62 \\
\hline Tonifying and replenishing medicinal & Heat-clearing and detoxicating & 57.11 & 98.54 \\
\hline Tonifying and replenishing medicinal & Wind-dampness dispelling medicinal & 79.97 & 97.99 \\
\hline Blood-activating and stasis-resolving medicinal & Wind-dampness dispelling medicinal & 79.96 & 97.92 \\
\hline Tonifying and replenishing medicinal & Blood-activating and stasis-resolving medicinal & 94.59 & 97.80 \\
\hline
\end{tabular}

Table 3: The top 9 kinds of function herb among the 332 herbs of the formulae in frequency.

The top three pairs of herbal functions with the highest confidence included the tonifying and replenishing medicinal with waterdischarging and damp-clearing medicinal (98.62\%), tonifying and replenishing medicinal with heat-clearing and detoxicating medicinal (98.54\%), and tonifying and replenishing medicinal with wind- dampness dispelling medicinal (97.99\%) (Table 3). Occurrence frequency=number of occurrences for the herbs appearing in various formulae/total cumulative occurrences for 332 herbs appearing in 3609 formulae; Frequency of use=number of formulae recording the use of the herbs/total number of selected formulae. 
Page 6 of 8

\section{Associations between pairs of couplet herbs from the formulae}

Couplet herbs are two herbs used in pair to increase the therapeutic effect or reduce the toxic effect. To further examine the compatibility patterns of couplet medicinal prescriptions, e targeted the herbs for blood-activating and stasis-resolving, tonifying and replenishing, and the herbs for water-disinhibiting damp-percolating medicina, heatclearing and detoxicating and wind-dampness dispelling medicinal, which were frequently prescribed for the treatment of osteoarthritis (Table 2). The minimum support of 0.2 and the minimum confidence of 0.7 were specified. The top three pairs of couplet herbs with the highest confidence included the Hong Hua paired with Tao Ren (98.90\%), he Pu Gong Ying paired with Bai Hua She Cao (97.88\%), and the Fu Ling paired with Ze Xie (92.63\%) (Table 4).

\begin{tabular}{|l|l|l|l|}
\hline After item & Befor item & Support (\%) & Confidence (\%) \\
\hline Hong Hua & Tao Ren & 68.41 & 98.90 \\
\hline Pu Gong Ying & Bai Hua She She Cao & 38.01 & 97.88 \\
\hline Fu Ling & Ze Xie & 48.10 & 92.63 \\
\hline
\end{tabular}

\begin{tabular}{|l|l|l|l|}
\hline Fu Ling & Xi Xian Cao & 43.25 & 91.99 \\
\hline Fu Ling & Shan Yao & 72.18 & 90.78 \\
\hline Fu Ling & Pu Gong Ying & 56.30 & 90.55 \\
\hline Fu Ling & Tao Ren & 68.41 & 90.23 \\
\hline Fu Ling & Yi Yi Ren & 69.16 & 89.30 \\
\hline
\end{tabular}

Table 4: The commonly used pairs of couplet herbs in the formulae.

\section{Associations between herbs and Immune Inflammation and Metabolic Index from the formulae}

The minimum support of 0.2 and the minimum confidence of 0.7 were specified. Du Huo and Chen Pi was associated with increased super oxide dismutase enzyme(77.40\%). Mai Ya and Dan Sheng was associated with decreased high-sensitivity C-reactive protein, erythrocyte sedimentation rate, complement C3, complement C4, g(A). Mai Ya and Fu Ling was associated with decreased IgM (74.37\%) (Table 5)

\begin{tabular}{|c|c|c|c|c|}
\hline After item & \multicolumn{2}{|l|}{ Before item } & Support (\%) & Confidence (\%) \\
\hline Du Huo, hen Pi & Metabolic index & $\mathrm{SOD} \uparrow$ & 28.79 & 77.40 \\
\hline Mai Ya, an Sheng & \multirow[t]{2}{*}{ Inflammation index } & hs-CRP $\downarrow$ & 28.61 & 75.25 \\
\hline Mai Ya, an Sheng & & $\mathrm{ESR} \downarrow$ & 27.88 & 70.72 \\
\hline Mai Ya, an Sheng & \multirow[t]{5}{*}{ Immune index } & Complement C3 $\downarrow$ & 28.67 & 75.70 \\
\hline Mai Ya, an Sheng & & Complement C4 $\downarrow$ & 28.68 & 77.69 \\
\hline Mai Ya, an Sheng & & $\lg A \downarrow$ & 28.67 & 75.59 \\
\hline Mai Ya, an Sheng & & $\lg G \downarrow$ & 28.65 & 75.68 \\
\hline Mai Ya, u Ling & & $\lg M \downarrow$ & 30.67 & 74.37 \\
\hline
\end{tabular}

Table 5: Correlation of TCM with immune inflammation and metabolic index.

\section{Discussion}

According to the traditional Chinese medicine. Osteoarthritis belongs to the category known as Bi Syndrome in traditional Chinese medicine terminology. Based on traditional Chinese medicine theories, efficiency of spleen qi and inadequate source of engendering transformation is internal cause. Wind, old, dampness, rot, dryness and other pathogen is external cause, which invading the human body and blockings channels. These are believed to be the main mechanism responsible for development of osteoarthritis.

\section{Medicinal formulae often include herbs that are warm or cold}

The 332 herbs were classified according to the Five Properties, and herbs that were warm or cold were the top two most frequently prescribed herbs in the formulae. In TCM theories, herbs with a warm have effect of clearing heat-fire. Herbs with a cold have effect of expelling cold from channel.

\section{Medicinal formulae often include herbs that are sweet or bitter}

The 332 herbs were classified according to the Five Flavors, and herbs that were sweet or bitter were the top two most frequently prescribed herbs in the formulae. In TCM theories, herbs that taste sweet can be used for mediation, tonification and laxation. They are mainly used for deficiency syndrome and pain syndrome in clinical. Herbs that taste bitter can be used for purgation, purging fire, directing qi downward and dried dampness. They are mainly used in the treatment of various dampness-heat syndrome and cold-dampness syndrome in clinical. However, wet tasting herbs with spleen strengthening functions were prescribed and used more frequently than herbs with a bitter taste for clearing heat.

There is a close relationship between osteoarthritis and spleen, and herbs for replenishing qi and fortifying the spleen, blood-activating and stasis-resolving, dry dampness to fortify the spleen, heat-clearing and detoxicating are the keys of osteoarthritis treatment

Spleen deficiency is the main pathogen in the pathogen of osteoarthritis. Previous experimental studies have proved that the 
effect of traditional Chinese medicine spleen unit therapy in improving the patient of osteoarthritis with quality of life, Cardiopulmonary function and other aspects is outstanding. Animal experiments for Chinese medicine spleen unit treatment of knee osteoarthritis immunological mechanisms were explored [21]. Deficiency of spleen qi will cause endogenous dampness; deficiency of spleen qi cannot transport qi and blood; disorder of qi and blood can't nourish the muscles and limbs; deficiency of spleen qi will cause driving weakness and blood stasis. Both phlegm and blood stasis caused by each other; deficiency of qi and qi, efficiency of spleen qi will make dysfunctional of organs. The above can lead Bi syndrome. Therefore, reinforcing the healthy qi and eliminating the pathogenic factors is the key factor in treating osteoarthritis. Reinforcing the healthy qi is given priority to with strengthening spleen[22]. Because deficiency of spleen qi can cause dampness evil endogenous and make Bi Syndrome occurrence, aggravation or relapse. So, therapies give priority to dry dampness to fortify the spleen, combining with promoting blood circulation to remove meridian obstruction, spelling wind and eliminating dampness, and clearing away heat and toxic materials. Therefore, he herbs that have high affinity to the spleen channel were used frequently (frequency of use $=35.24 \%$ ), appeared 3608 times (99.97\%).

\section{The use of replenishing qi and fortifying the spleen herbs is the basis of prescription patterns for treatment of osteoarthritis}

Restoration of healthy qi is an effective way to treat diseases and to prevent further progression. The use of qi tonifying and spleenfortifying herbs is to replenish the source of engendering transformation for qi and blood, and to achieve qi-tonifying, bloodreplenishing and harmony of the five visceral functions. This is particularly essential for nourishing the liver and smoothing the qi movement. At the same time, spleen-strengthening and qireplenishing herbs also have the functions for resolving dampness and dispelling phlegm. Therefore, the formulae prescribed herbs such as Huang Qi, Bai Zhu, and Fu Ling, among others. While not forgetting the application of eliminate the pathogenic factors medicine. Phlegm and blood stasis is the pathogenesis of pathogenic factors in the body of the product, but also the cause of the body disease. Therefore, the formulae prescribed herbs such as Yi Yi Ren, Fu Ling, and Chen Pi eliminate phlegm. Tao Ren, ong Hua and Niu Xi disperse blood stasis and dredge collateral. Pu gong ying and Bai Hua She She Cao clear away heat and toxic materials. From the association rules mining, he results showed that the combination of the herbs should also focus on the functions for reinforce the healthy qi and eliminate the pathogenic factors. The use of couplet herbs involving Fu Ling and $\mathrm{Ze} \mathrm{Xie} \mathrm{is} \mathrm{to}$ achieve the effects of spleen-strengthening and qi-replenishing, and dampness-drying and water-draining; the use of couplet herbs involving Tao Ren and Hong Hua is to achieve the effects of promoting blood circulation to remove blood stasis; the use of couplet herbs involving Pu Gong Yin and Bai Hua She Cao is to achieve the effects of clearing away heat and toxic materials.

The correlation analysis between herbs and immuno-inflammatory and metabolic indexes showed that Mai Ya, an Sheng and Fu Ling had the highest correlation with inflammatory markers such as hypersensitive $\mathrm{C}$-reactive protein, erythrocyte sedimentation rate, complement C3, complement C4, immunoglobulin (A). Du Huo and Chen Pi had the highest correlation with superoxide dismutase (SOD). Mai Ya are used directly for strengthening spleen. Dan Sheng is used directly for promoting blood circulation to remove blood stasis. Fu
Ling can strengthen spleen and clear damp and promoting diuresis. The level of ESR, hs-CRP, complement C4, complement C3 can be reduced, and SOD can be increased, which thus (may) reduce the immune inflammatory response in patients with osteoarthritis, relieve joint symptoms and joint pain. Past studies have shown that Dan Sheng could significantly increase the peritoneal macrophages and phagocytic clearance capacity, enhanced the immune system of mice [23].

\section{Conclusions}

The results showed that treatment of osteoarthritis is considered to have a close relationship with spleen dysfunctions. These prescriptions focus on the herbs for replenishing qi and fortifying the spleen, and promoting blood circulation to remove blood stasis, and invigorating spleen for eliminating dampness and clearing away heat and toxic materials. Strengthening spleen seems to be the key to successful treatment. Meanwhile, the use of tonifying and replenishing and spleen-strengthening herbs also forms the basis of prescription patterns.

\section{References}

1. Chinese Medical Association (2013) Osteoarthritis. Rheumat Arthritis 2: 71-72.

2. Cheng ZH, Qunjie Z (2016) The epidemiological investigation of the status quo of osteoarthritis of the knee in China. Guangdong Medical Science 37.

3. Ministry of Health Statistical Information Center (2004) China health service survey-third national health service analysis and analysis. Beijing: Peking Union Medical College Press pp: 25-27.

4. Mannoni A, Riganti MP, Bari M, Ferrucci L, Costanzo S, et al. (2003) Epidemiological profile of symptomatic osteoarthritis in older adults: A population based study in Dicomano, Italy Ann Rheum Dis 62: 576-578.

5. Liu W (2009) Integrative Medicine Rheumatism Immunology. Wuhan: Huazhong University of Science and Technology Press 11, China.

6. Zhang W, Wang H, Zhang R, Zhang Y, Li X, et al. (2016) Evidence of Chinese herbal medicine Duhuo Jisheng decoction for knee osteoarthritis: A systematic review of randomized clinical trials. BMJ Open 6:e08973.

7. Nie B, Li X, Wei Y, Chen M, Zhou J, et al. (2006) Xianfanghuomingyin, Chinese compound medicine, modulates the proliferation and differentiation of $\mathrm{t}$ lymphocyte in a collagen-induced arthritis mouse model. Evid Based Complement Altern Med.

8. Li-ping R, Jian L, Wen-fang E (2015) Effects of TCM unit therapy of invigorating spleen on life quality and cardiopulmonary Function of patients with knee osteoarthritis and its immunological mechanism. Rheuma Arthritis. 4: 5-11.

9. Cheng YY, Liu J, Feng YX, Liu L, Wan L (2012) Xinfeng Capsule improves cardiopulmonary function of knee osteoarthritis rats by BTLA-HVEM signaling pathway to induce Treg immune tolerance. Chin J Cell Mol Immuno 28: 1133-1137.

10. Cheng YY, Liu J, Lei W, Jinshan Z, Yunxia F, et al. (2013) The effects of XinFeng capsule on $\mathrm{B}$ and $\mathrm{T}$ lymphocyte attenuator and oxidative stress of knee osteoarthritis patients. J Immunol 29: 416-421.

11. Bin T, Jian L, Heng ZP (2016) Mechanism exploration on osteoarthritis with blood stasis improved by Xinfeng capsule based on the NF-B signaling pathway. World J Integr Trad Western Med 11: 1274-1281.

12. Agrawal R, Mielinski T, Swami A (1993) Mining Association rules between sets of items in large databases int cof management of data C:ACM Press, Ash Washington USA pp: 207-216.

13. Bingru Y, Sun H, Xiong F (2002) Mining antitative association rules with standard SQL queries and it's evaluation. J Comp Res Dev 39: 307-312. 
Citation: Qiao Z, Jian L, Ling X, Qian S, Jin-chen G, et al. (2017) Exploratory Compatibility Regularity of Chinese Medicinal Prescription in Treating Osteoarthritis . Altern Integr Med 6: 244. doi:10.4172/2327-5162.1000244

Page 8 of 8

14. Shang Erxin, an Xinsheng, uan Jinjuan, $t$ al. (2010) Data mining study on incompatibility characters of Chinese herbal medicine in accordance with association rules. J Nanjing University Trad Chinese Med 26.

15. Chinese Medical Association of Rheumatology Professional Committee (2003) Osteoarthritis diagnosis and treatment guidelines. Chin J Rheumatol 7: 702-704.

16. Nanjing University of Traditional Chinese Medicine (2006) Dictionary Shanghai: Shanghai Science and Technology Press, China.

17. Xuemin G, Yongyan Z (2007) Chinese medicine. Beijing: Chinese Medicine Publishing House 1, China.

18. Yu Yi, Chen Yi, Zhonglin Z (2014) Improvement and realize of Apriori algorithm. Microcomputer and Applications 23: 10-12.

19. Yu S, Xiao-li E, Yuan HG (2013) Application and simulation of association rules in medical cloud data orientation. Comp Simul 30: 239-242.
20. YU Hai-bin, Su-yun I, Liang EJ (2013) Intelligent integration and application of TCM clinical medical research information sharing system. World Sci Tech-Mod Trad Chin Med 15: 1480-1414.

21. Li-ping R, Jian Liu, Fang EW (2015) Effects of TCM unit therapy of invigorating spleen on life quality and cardiopulmonary function of patients with knee osteoarthritis and its immunological mechanism. Rheumat Arthritis 4: 5-11.

22. Cheng Yuan-yuan, Jian L, Lei X (2013) Study on the relationship between osteoarthritis and spleen deficiency. Chin J Basic Med Trad Chin Med 19: 742-749.

23. Qiangu L, Jiuyao Z, Yuqi I (2011) Effect of membranaceus and salvia decoction on the immune system of mice. China Medical Herald 8: 23-24. 